\title{
Fatores associados ao desenvolvimento de hipertensão arterial em uma comunidade quilombola*
}

Factors associated with the development of arterial hypertension in a quilombola community

Factores asociados con el desarrollo de hipertensión arterial en una comunidad quilombola

\section{Dinah Alencar Melo AraújoI, Tatiana Victoria Carneiro Moura ${ }^{\mathrm{II}}$, Denival Nascimento Vieira Júnior ${ }^{\mathrm{III}}$, Francisco João de Carvalho Neto ${ }^{\mathrm{IV}}$, José de Siqueira Amorim Júnior ${ }^{\mathrm{V}}$, Ana Roberta Vilarouca da Silva ${ }^{\mathrm{VI}}$}

\begin{abstract}
Resumo: Objetivo: avaliar os fatores associados ao desenvolvimento da Hipertensão Arterial (HA) em indivíduos residentes de uma comunidade quilombola. Método: estudo transversal, realizado com 74 participantes. Os dados foram coletados por meio do preenchimento de dois formulários, sendo eles: sociodemográfico/socioeconômico e sobre estilo de vida. Realizou-se análise estatística descritiva e inferencial. Resultados: predominou o sexo feminino, raça preta, casados, ensino fundamental incompleto e a classe econômica C2. 37,8\% autorreferiu presença da HA, destes, $22,9 \%$ eram mulheres e $14,9 \%$, homens. Obteve-se maiores percentuais de não obesos, não tabagistas, não alcoólatras e praticantes de algum tipo de atividade física. Quanto aos hábitos alimentares, observou-se o consumo de frutas, legumes, hortaliças, temperos industrializados, alimentos ultraprocessados e processados. Quanto as recomendações sobre alimentação saudável, 64,9\% afirmaram nunca ter recebido orientações e 48,6\% não procura o serviço de saúde. Conclusão: foi constatado percentuais significativos relativos a fatores de risco para o desenvolvimento da hipertensão.
\end{abstract}

\footnotetext{
${ }^{\text {I }}$ Enfermeira pela Universidade Federal do Piauí. Picos, Piauí, Brasil. E-mail: jcamo@hotmail.com, Orcid: https://orcid.org/0000-0003-0922-349X.

II Enfermeira, mestre em Ciências e Saúde pela Universidade Federal do Piauí. Picos, Piauí, Brasil. E-mail: tatimourafe@gmail.com, Orcid: http://orcid.org/0000-0001-9214-6012.

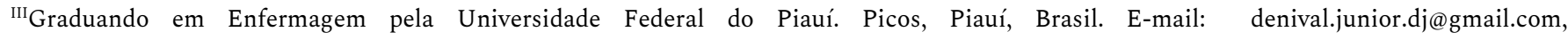
https://orcid.org/0000-0001-8813-0472.

IV Graduando em Enfermagem pela Universidade Federal do Piauí. Picos, Piauí, Brasil. E-mail: franciscojoaodecarvalhoneto@gmail.com, Orcid: https://orcid.org/0000-0002-2011-5900.

v Enfermeiro, mestre em Ensino na Saúde pela Universidade Estadual do Ceará. Picos, Piauí, Brasil. E-mail: enf.juniorsiqueira@gmail.com, Orcid: https://orcid.org/0000-0002-3401-5417.

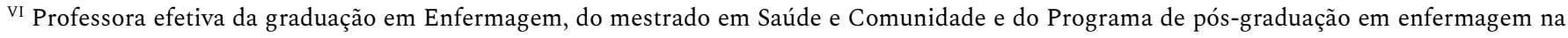
Universidade Federal do Piauí. Picos, Piauí, Brasil. E-mail: robertavilarouca@yahoo.com.br, Orcid: http://orcid.org/0000-0001-5087-4310.
}

*Extraído do Trabalho de conclusão do Curso de Graduação Bacharelado em Enfermagem, pela Universidade Federal do Piauí, Picos, 2019. 
Fatores associados ao desenvolvimento de hipertensão arterial em uma comunidade qui... | 2

Descritores: Hipertensão; Fatores de Risco; Grupo com Ancestrais do Continente Africano; Indicadores Básicos de Saúde; Epidemiologia

Abstract: Objective: to evaluate factors associated with the development of Arterial Hypertension (AH) among individuals living in a quilombola [peopled with ancestors from the African continent] community. Method: crosssectional study, carried out with 74 participants. Data were collected by filling out two forms with sociodemographic/ socioeconomic and lifestyle questions. Descriptive and inferential statistical analysis was performed. Results: female gender, black race, married, incomplete elementary education and economic class C2 predominated. The presence of AH was self-reported by $37.8 \%$, of these $22.9 \%$ were women and $14.9 \%$ men. Higher percentages of non-obese, non-smoking, non-alcoholic and practitioners of some type of physical activity were obtained. Regarding dietary habits, the consumption of fruit, vegetables, salads, industrialized spices, processed and ultra-processed foods was observed. Concerning the recommendations on healthy eating, $64.9 \%$ stated they had never received guidance and $48.6 \%$ did not seek health care. Conclusion: significant percentages were found relating to risk factors for the development of AH.

Descriptors: Hypertension; Risk Factors; Group with Ancestors from the African Continent; Health Status Indicators; Epidemiology

Resumen: Objetivo: evaluar los factores asociados al desarrollo de la Hipertensión Arterial (IA) en personas residentes en una comunidad quilombola. Método: estudio transversal, realizado con 74 participantes. Los datos se recogieron rellenando dos formularios, sociodemográfico/socioeconómico y de estilo de vida. Se realizó un análisis estadístico descriptivo y de inferencia. Resultados: predominó el sexo femenino, la raza negra, los casados, la educación básica incompleta y la clase económica C2. El 37,8\% auto declaró la presencia de AH, de los cuales el $22,9 \%$ eran mujeres y el $14,9 \%$, hombres. Se obtuvieron mayores porcentajes de no obesos, no fumadores, no alcohólicos y practicantes de algún tipo de actividad física. En cuanto a los hábitos alimentarios, se observó el consumo de frutas, verduras, hortalizas, especias procesadas, alimentos ultra procesados y procesados. En cuanto a las recomendaciones sobre alimentación sana, el 64,9\% afirma no haber recibido nunca orientaciones y el 48,6\% no solicita el servicio de salud. Conclusión: se constataron porcentajes significativos relativos a los factores de riesgo para el desarrollo de la hipertensión.

Descriptores: Hipertensión; Factores de Riesgo; Grupo con Antepasados del Continente Africano; Indicadores de Salud; Epidemiología

\section{Introdução}

As doenças crônicas não transmissíveis (DCNT) caracterizam-se como um dos maiores problemas de saúde pública no mundo, ocorrendo em maior escala nos países subdesenvolvidos, onde há populações mais pobres e vulneráveis. Possuem evolução lenta, na maioria das vezes são assintomáticas, geram sofrimento, complicações irreversíveis e impactos econômicos. A Hipertensão Arterial (HA) se insere nesse grupo, configurando-se como uma das principais causas de adoecimento e óbito. A HA consiste em um problema circulatório com elevada prevalência, 
baixa taxa de controle e alto poder incapacitante. É considerada um dos principais fatores de risco para o desenvolvimento de doenças cardiovasculares (DCV), renais, encefálicas e vasculares. ${ }^{1}$

Os fatores de risco para o desenvolvimento da HA abrangem alimentação inadequada, alcoolismo, tabagismo, inatividade física, excesso de peso, distúrbios do metabolismo da glicose e lipídios, além de fatores étnicos, ambientais e socioeconômicos. ${ }^{2-3}$ No que tange o fator étnico, a raça negra possui duas vezes mais chances de desenvolver a HA do que indivíduos brancos, uma possível explicação está na teoria de que os negros apresentariam um gene economizador de sódio que leva ao influxo celular de sódio e efluxo celular de cálcio, propiciando alterações orgânicas que resultam na elevação da pressão arterial (PA). ${ }^{4}$

De acordo com as evidências científicas a situação de saúde tem sido relacionada com questões étnico-raciais, revelando desigualdades nos perfis de adoecimento e morte ao se comparar populações brancas com as que se autodeclaram pretos e pardos. Esses grupos se encontram em situação de vulnerabilidade social e sofrem com as disparidades na oferta de serviços de saúde, enfrentando dificuldades no acesso, tornando-os mais vulneráveis a doenças como a HA. ${ }^{5}$

É nesse contexto que se insere as comunidades quilombolas, cuja definição baseia-se em critérios de autoatribuição, com trajetória histórica própria, vínculos territoriais específicos e presunção na ancestralidade negra relacionada com a resistência à opressão histórica sofrida. Localizam-se, geralmente, em áreas rurais, possuindo altos índices de analfabetismo e apresentando precárias condições de vida, moradia, saneamento e acesso aos serviços de saúde. ${ }^{6}$

Partindo do princípio de que a HA é uma condição clínica multifatorial, sendo na maioria dos casos um resultado final da interação entre fatores genéticos, socioculturais, ambientais e demográficos, ${ }^{2-3}$ a presente pesquisa busca responder a seguinte questão de pesquisa: quais os principais fatores que contribuem para o desenvolvimento da HA em residentes de comunidade quilombola no Piauí (PI)? 
Fatores associados ao desenvolvimento de hipertensão arterial em uma comunidade qui... $\mid 4$

Torna-se imperioso retratar essa população por serem agrupamentos étnicos em situação de vulnerabilidade social e que, historicamente, passaram por um processo de expropriação de cultura e direitos resultando em impactos nos indicadores de saúde. Além disso, são raras as pesquisas que buscam conhecer a situação de saúde e os determinantes dos agravos que atingem populações vulneráveis. Dessa forma, os estudos acerca da temática possibilitam a compreensão dos condicionantes do processo saúde/doença nessas populações, além de contribuir para a formulação e efetivação de políticas públicas. Diante disso, o presente estudo teve como objetivo avaliar os fatores associados ao desenvolvimento da HA em indivíduos residentes de uma comunidade quilombola.

\section{Método}

Estudo transversal realizado em uma comunidade quilombola localizada na região centro-sul do Estado do PI, Brasil. A comunidade está localizada a sete quilômetros da sede da cidade de Paquetá - PI, possui autodefinição de sua identidade étnico racial e é certificada junto à Fundação Cultural Palmares.

A população constituiu-se de moradores da comunidade, a qual é composta por 48 famílias cadastradas na Estratégia de Saúde da Família (ESF), 167 moradores. Como critérios de inclusão adotou-se: ter idade acima de 18 anos; ser residente da comunidade quilombola; participar de todas as etapas da pesquisa, incluindo a entrevista, a mensuração das medidas antropométricas e aferição da PA; ser cadastrado e acompanhado pela ESF. E como critérios de exclusão: impedimento para obtenção das medidas antropométricas, por exemplo, ser cadeirante ou acamado; gestantes, devido à ocorrência de alteração no Índice de Massa Corporal (IMC) e pessoas com déficit cognitivo informado pelo participante ou familiar. Foram excluídos 93 moradores, sendo a amostra constituída por 74 participantes. 
5 | Araújo DAM, Moura TVC, Júnior DNV, Neto FJC, Júnior JSA, Silva ARV

Inicialmente, entrou-se em contato com o líder comunitário para a explicação e concedimento de autorização para a realização deste projeto. Em seguida, contactou-se a enfermeira responsável pela ESF que assiste a comunidade, para que conjuntamente com as Agentes Comunitárias de Saúde (ACS) fosse possível organizar um cronograma.

A coleta dos dados ocorreu no próprio domicílio do participante, durante os meses de setembro e outubro de 2019. Na ocasião, o pesquisador apresentou o projeto e explicou os objetivos da pesquisa, riscos e benefícios. Os que concordaram em participar assinaram o termo de consentimento livre e esclarecido e, em seguida, aplicou-se dois formulários, um contendo dados socioeconômico e sociodemográfico e outro com variáveis relacionadas ao estilo de vida e aos dados antropométricos, os quais estavam inseridos no aplicativo EpiCollect. O aplicativo foi utilizado pela pesquisadora e equipe previamente treinada por ela, totalizando cinco membros.

Nas residências compostas por mais de dois moradores, realizou-se a aplicação dos formulários individualmente em ambientes distintos para minimizar o risco de constrangimento, tendo a atividade realizada dentro de, aproximadamente, 15 minutos. O formulário socioeconômico e sociodemográfico buscou coletar informações referentes a idade, sexo, raça, situação conjugal, escolaridade, composição e renda familiar, presença de HA e tempo estimado de diagnóstico. Após essas informações, realizou-se a classificação econômica, definida pelas classes A, B1, B2, C1, C2, D, E.

Quanto as variáveis sobre estilo de vida, essas se subdividiram em antropométricas, hábitos de vida, alimentares e valores da PA. O critério utilizado para identificação racial foi a autoatribuição, conforme definido pelo Instituto Brasileiro de Geografia e Estatística (IBGE).

Quanto as variáveis antropométricas, a verificação do peso deu-se por meio de uma balança digital portátil, a qual foi instalada em local nivelado. Após a balança ser ligada foi verificado se estava zerada. Em seguida, o participante foi instruído a se colocar no centro da balança, descalço, ereto, com os pés juntos e os braços estendidos ao longo do corpo.7 
Fatores associados ao desenvolvimento de hipertensão arterial em uma comunidade qui... $\mid 6$

Como todos os participantes da pesquisa deambulavam, a estatura foi verificada com o indivíduo em pé, por meio de uma fita métrica não flexível de 1,5 metros, a qual foi fixada em parede plana, a $100 \mathrm{~cm}$ do solo. O participante foi orientado a ficar descalço, retirar adereços da cabeça, posicionar-se no centro do local com a fita, com os pés o mais próximo possível da parede e formando um anglo de $90^{\circ}$ com as pernas, braços entendidos ao longo do corpo e com o olhar para um ponto fixo ao nível dos olhos. ${ }^{7}$

Com o intuito de avaliar o estado nutricional adotou-se o IMC, o qual é obtido por meio da razão entre o peso e o quadrado da altura. Classificou-se como baixo peso os resultados menores ou igual a $22 \mathrm{~kg} / \mathrm{m}^{2}$, adequado ou Eutrófico entre 22 e $27 \mathrm{~kg} / \mathrm{m}^{2}$ e sobrepeso acima ou igual a $27 \mathrm{~kg} / \mathrm{m} .^{2,7}$

A fim de avaliar a adiposidade central, um marcador que identifica possíveis riscos cardiovasculares e metabólicos, realizou a mensuração da circunferência da cintura (CC). Após obtenção do valor foi classificado como obesos os participantes que obtiverem CC >102 $\mathrm{cm} \mathrm{e}$ obesas as participantes que obtiverem $\mathrm{CC}>88 \mathrm{~cm} .^{8}$

Para a obtenção da PA, seguiu-se a técnica da VII Diretriz Brasileira de Hipertensão Arterial, sendo considerado como pré-hipertensão os valores entre 121/81 a 139/89; hipertensão estágio I entre 140/90 a 159/99; hipertensão estágio II entre 160/100 a 179/109 e hipertensão estágio III os valores $\geq$ 180/110. Os valores foram computados e corresponderam a Pressão Arterial Média (PAM). ${ }^{8}$ Com relação às variáveis de hábitos de vida e alimentares buscou-se coletar informações acerca dos padrões de consumo alimentar individual e comportamentos desenvolvidos pelos indivíduos.

Os dados coletados foram digitados e analisados por meio do programa estatístico IBM Statistical Package for the Social Sciences (SPSS), versão 20.0. Para análise dos dados utilizou-se o cálculo da média, desvio padrão, valores máximos e mínimos, além do Teste Qui-quadrado de Pearson, Teste de Coeficiente de correlação de Spearman, Teste U-Mann-Whitney e KruskalWallis. Para o teste de correlação de Spearman considerou-se os seguintes valores para a associação: 0 (nula); 0-0,3 (fraca); 0,3-0,6 (regular); 0,6-0,9 (forte); 0,9-1 (muito forte) e 1 (perfeita). 
Os achados foram apresentados por meio de tabelas, discutidos e analisados de acordo com a literatura pertinente à temática. Para todas as análises estatísticas inferenciais foram consideradas como estatisticamente significantes aquelas com $\mathrm{p}<0,05$.

O projeto foi aprovado no dia 15 de agosto de 2019, pelo Comitê de Ética em Pesquisa da Universidade Federal do Piauí, sob parecer nº 3.511.138/CAAE: 18501519.8.0000.8057, seguindo todos os padrões ético exigidos pelas Resoluções 466/2012 - 510/2016 - 580/2018, do Ministério da Saúde. A pesquisadora responsável seguiu todos os princípios bioéticos como a beneficência, não maleficência, autonomia e justiça.

\section{Resultados}

Foram analisados 74 residentes da comunidade quilombola, o sexo predominante foi o feminino (55,4\%). Em relação à idade, variou entre 18 e 78 anos, com média de 44,2 anos e desvio padrão de 17,6. Reportando-se à raça, predominou os que se autodenominaram pretos $(67,6 \%)$. Em relação à situação conjugal, $55,4 \%$ eram casados e moravam com os familiares $(45,9 \%)$. No que tange a escolaridade, predominou-se o ensino fundamental incompleto $(37,8 \%)$ e quanto à classe econômica, prevaleceu a classe C2 (39,2\%).

Relacionado às variáveis antropométricas e ao estilo de vida, $50 \%$ afirmou não possuir HA, 12,2\% relatou não saber se possuíam e 37,8\% autorreferiram a presença da doença (destes $22,9 \%$ eram mulheres e $14,9 \%$ em homens). O tempo de diagnóstico da HA predominou entre um e cinco anos de presença da morbidade $(16,2 \%)$.

No que se refere à PA, 39,2\% apresentaram valores pressóricos normais. Relativo ao IMC, $47,3 \%$ apresentou peso adequado, com média e desvio padrão de $24,63 \pm 3,95 \mathrm{~kg} / \mathrm{m}^{2}$. A CC variou entre $51,5 \mathrm{~cm}$ e $113,0 \mathrm{~cm}$, predominando-se a classificação de não obeso $(67,5 \%)$. Quanto ao tabagismo, predominou não fumante $(89,2 \%)$, assim como os que não consumiam bebida 
\begin{tabular}{ll|l} 
Fatores associados ao desenvolvimento de hipertensão arterial em uma comunidade qui... & 8
\end{tabular}

alcoólica (54,1\%). Ainda, mais da metade dos participantes referiu a prática de algum tipo de exercício físico (52,7\%).

Quando indagados sobre os seus hábitos alimentares, $62,2 \%$ realizavam três refeições ao dia, 91,9\% consumiam frutas, 95,9\% legumes e hortaliças e 73\% referiu o uso de temperos industrializados, entretanto, 94,6\% afirmou não acrescentar sal à comida pronta. Relativo aos embutidos ou enlatados, $54,1 \%$ não consome esse tipo de alimento, contudo mais da metade dos participantes relatou o consumo de bolacha/biscoitos, salgados fritos e ingesta de refrigerantes (respectivamente 79,7\%, 56,8\% e 68,9\%). No que concerne às recomendações sobre alimentação saudável, 64,8\% afirmaram nunca ter recebido essas orientações e 48,6\% não procura o serviço de saúde por causa da HA.

A Tabela 1 aponta a distribuição numérica e percentual da classificação da PA segundo a faixa etária. Observou-se que $22 \%$ dos participantes com faixa etária entre 18 e 25 anos de idade estão enquadrados na classificação de normal ou pré-hipertensão. A hipertensão de estágio I prevaleceu os grupos entre 34 a 41 e 58 a 65 anos, com 4\% cada grupo. A HA de estágio II predominou entre as idades 66 a 80 anos, com 5,4\%. Na hipertensão de estágio III, houve um participante na faixa etária entre 66 e 72 anos $(1,3 \%)$.

Tabela 1 - Distribuição numérica (n) e percentual (\%) da classificação da pressão arterial segundo a faixa etária $(\mathrm{n}=74)$. Picos, 2019.

\begin{tabular}{|c|c|c|c|c|c|c|c|c|}
\hline \multirow[b]{2}{*}{$\begin{array}{c}\text { Faixa etária } \\
\text { (em anos) }\end{array}$} & \multicolumn{2}{|c|}{$\begin{array}{c}\text { Normal ou pré- } \\
\text { hipertensão }\end{array}$} & \multicolumn{2}{|c|}{ HA I* } & \multicolumn{2}{|c|}{$\mathrm{HA} \mathrm{II}^{+}$} & \multicolumn{2}{|c|}{ HA III ${ }^{\ddagger}$} \\
\hline & $\mathrm{n}$ & $\%$ & $\mathbf{n}$ & $\%$ & $\mathbf{n}$ & $\%$ & $\mathbf{n}$ & $\%$ \\
\hline $18-25$ & 16 & 22,0 & 1 & 1,3 & - & - & - & - \\
\hline $26-33$ & 3 & 4,0 & - & - & - & - & - & - \\
\hline $34-41$ & 10 & 13,5 & 3 & 4,0 & - & - & - & - \\
\hline $42-49$ & 7 & 9,5 & 2 & 2,7 & 1 & 1,3 & - & - \\
\hline $50-57$ & 12 & 16,3 & 1 & 1,3 & 1 & 1,3 & - & - \\
\hline $58-65$ & 2 & 2,7 & 3 & 4,0 & - & - & - & - \\
\hline $66-72$ & 2 & 2,7 & 1 & 1,3 & 2 & 2,7 & 1 & 1,3 \\
\hline $73-80$ & 3 & 4,0 & 1 & 1,3 & 2 & 2,7 & - & - \\
\hline
\end{tabular}

${ }^{*}$ Hipertensão Arterial estágio I. ${ }^{+}$Hipertensão Arterial estágio II. ${ }^{*}$ Hipertensão Arterial estágio III. p-Valor $=0,000$ 
9 | Araújo DAM, Moura TVC, Júnior DNV, Neto FJC, Júnior JSA, Silva ARV

$\mathrm{Na}$ Tabela 2, foi relacionado à variável possui HA com as variáveis relacionadas ao estilo de vida e alimentação. Houve associação estatística significativa entre a variável avaliada e receber ou não recomendações sobre a alimentação $(\mathrm{p}=0,002)$ evidenciando que não ser orientado sobre uma alimentação adequada, pode favorecer ao surgimento de HA.

Tabela 2 - Associação entre a variável possui HA com as variáveis relacionadas ao estilo de vida e alimentação dos participantes (n=74). Picos-PI, 2019.

\begin{tabular}{cc}
\hline & Possui hipertensão arterial \\
\cline { 2 - 2 } Variáveis qualitativas & p-Valor \\
\hline Atividade física & 0,386 \\
Tabagismo & 0,277 \\
Recomendações sobre alimentação & 0,002 \\
Consumo de alimentos embutidos & 0,683 \\
\hline
\end{tabular}

*Teste Qui-quadrado de Pearson.

Prosseguindo, realizou-se a análise entre a correlação entre a pressão arterial sistólica média (PADM) e pressão arterial diastólica média (PADM) com o IMC e CC, havendo correlação moderada entre o IMC e a PASM e baixa com a PADM $(r=0,350 ; p=0,002$ e $r=0,295 ; p=0,011)$ respectivamente. Além da CC correlacionar-se moderadamente com a PASM $(r=0,359 ; p=0,002)$ e correlação fraca com a PADM (r=0,282; $\mathrm{p}=0,015)$ (Tabela 3$)$.

Tabela 3 - Correlação entre e a média da pressão arterial sistólica e diastólica com o IMC e CC dos participantes (n=74). Picos-PI, 2019.

\begin{tabular}{ccc}
\hline Variáveis antropométricas & PASM $^{+}$ & PADM $^{*}$ \\
\hline IMC $\$$ & $r=0,350 ; p-0,002^{*}$ & $r=0,295 ; p-0,011^{*}$ \\
CC $\|$ & $r=0,359 ; p-0,002^{*}$ & $r=0,282 ; p-0,015^{\star}$ \\
\hline
\end{tabular}

"Coeficiente de correlação de Spearman. " Pressão Arterial Sistólica Média. ' Pressão Arterial Diastólica Média. sIMC. "|CC.

Ainda, foi realizada associação estatística entre variáveis socioeconômicas e sociodemográficas com a PASM e a PADM. Houve associação entre o sexo e a PASM (p=0,043). 
Fatores associados ao desenvolvimento de hipertensão arterial em uma comunidade qui... | 10

Em seguida, foi realizado teste de correlação de Spearman, havendo força de associação fraca entre a idade e a PASM $(r=0,298 ; \mathrm{p}=0,010)$ e correlação alta entre a renda familiar com a PADM $(r=0,808 ; p=0,0001)$. Encontrou-se associação estatística significativa entre a situação conjugal e a PASM e PADM (p- 0,029; 0,049) respectivamente (Tabela 4).

Tabela 4 - Associação entre a média da pressão arterial sistólica e diastólica com as variáveis socioeconômicas e sociodemográficas (n=74). Picos-PI, 2019

\begin{tabular}{ccc}
\hline Variáveis & PASM $^{\S}$ & PADM $^{\|}$ \\
\hline Sexo & $0,043^{*}$ & $0,240^{*}$ \\
Idade & $\mathrm{r}=0,298 ; \mathrm{p}=0,010^{+}$ & $\mathrm{r}=0,152 ; \mathrm{p}=0,197^{+}$ \\
Renda & $\mathrm{r}=1,000 ; \mathrm{p}=0,905^{+}$ & $\mathrm{r}=0,808 ; \mathrm{p}=0,0001^{+}$ \\
Raça & $0,079^{\ddagger}$ & $0,359^{\ddagger}$ \\
Situação conjugal & $0,029^{\ddagger}$ & $0,049^{\ddagger}$ \\
Escolaridade & $0,123^{\ddagger}$ & $0,184^{\ddagger}$
\end{tabular}

${ }^{*}$ Teste U-Mann-Whitney. ${ }^{\dagger}$ Coeficiente de correlação de Spearman. ${ }^{\ddagger}$ Kruskal-Wallis. §Pressão Arterial Sistólica Média. || Pressão Arterial Diastólica Média.

\section{Discussão}

Os resultados deste estudo revelaram um perfil populacional semelhante ao encontrado em diversas comunidades quilombolas do Brasil, composta por uma população com média de idade de 43 anos, predominantemente da raça preta, prevalência do sexo feminino, ${ }^{3} \operatorname{casados}^{5,9} \mathrm{e}$ alta taxa de analfabetismo/fundamental incompleto. ${ }^{10}$

É relevante estudar a variável escolaridade, visto que ela pode influenciar no tipo de ocupação dos indivíduos e, consequentemente na renda familiar. ${ }^{11}$ Além disso, quanto mais elevado o grau de instrução, melhor o entendimento e acesso a informações, levando, assim, o indivíduo a ter uma maior preocupação com sua saúde e bem-estar, consequentemente gerando melhor estado de saúde e qualidade de vida.

Outra característica marcante foi a baixa classe econômica, calculada com base nas condições de moradia e vida. Diversos estudos demostraram que esse público tende a possuir baixa renda.,10,12-14 Tais circunstâncias ocorrem devido às condições aos quais foram submetidos 
ao longo da formação histórica da comunidade o que reflete em doenças e agravos à saúde provenientes do processo de marginalização a que estão expostos. ${ }^{11}$

Nesta investigação, a presença da hipertensão foi elevada, assemelhando-se aos percentuais encontrados na literatura, os quais variam entre $26 \%$ a $52,5 \% .^{10,13,15-16}$ Todavia, não se pode comparar essas prevalências, considerando a diversidade de métodos utilizados em outros estudos, principalmente quanto à amostragem populacional.

Quanto ao tempo de diagnóstico, os resultados obtidos foram contrários ao da pesquisa transversal realizada com população quilombola do estado de Sergipe, a qual demonstrou uma média de convivência com a HA de 9,59 anos. ${ }^{10}$ No entanto, comparar essa vertente com a das demais comunidades pode resultar em erro, uma vez que ela está condicionada a presença de vários outros fatores, como a presença e qualidade do serviço de saúde na comunidade propiciando um diagnóstico precoce; escolaridade, aumentando o nível de informação; meios de transporte, favorecendo o deslocamento até o serviço de saúde; e renda.

Relativo ao gênero, desse percentual que autorreferiu HA, mais da metade eram mulheres, implicando assim em uma associação estatística significativa entre as variáveis sexo e PASM, podendo-se, assim, afirmar a maior prevalência da doença no sexo feminino. Estudo transversal obteve resultado contraditório, demostrando maior influência do sexo masculino à HA. ${ }^{12}$

Da mesma maneira, estudo ecológico, ao comparar a prevalência de HA entre homens e mulheres moradores da comunidade de Agrovila, situada no estado do amazonas, identificou percentual de 23,31\% na população acima de 18 anos feminina, e de 5,93\% na masculina acima de 18 anos, o que representou um risco de 3,934 vezes maior de prevalência de hipertensão em mulheres do que em homens, fator que não deve ser justificado como coincidência. ${ }^{17}$ Em contrapartida, pesquisas realizadas com o mesmo tipo de grupo populacional em questão, não encontraram diferença estatística significativa entre os gêneros e a elevação dos níveis pressóricos. ${ }^{3,10,13}$ No que se refere à classificação da PA a maioria apresentou valores pressóricos 
normais; no entanto, a taxa de pré-hipertensos expressou-se um pouco elevada, o que chama a atenção para um possível aumento da ocorrência de hipertensão nessa comunidade, visto que indivíduos pré-hipertensos podem evoluir para HA.

Relativo ao IMC, observou-se que a maior parte da população estudada se encontrava dentro dos padrões da normalidade, apresentando baixa taxa de sobrepeso. Estes dados corroboram com o estudo transversal realizado na Comunidade Quilombola de Tomé Nunes-Bahia, que ao analisar a prevalência do excesso de peso e os fatores associados em 112 adultos membros de uma comunidade quilombola identificaram $66,1 \%$ de eutrófico e $27,7 \%$ com excesso de peso. ${ }^{9}$ Todavia, esses resultados diferem dos demais achados literários os quais obtiveram em seus estudos alto índice de sobrepeso/obesidade, autoconsumo de alimentos gordurosos e de sal.,32,14

Embora o cálculo do IMC seja a ferramenta mais utilizada em pesquisas para classificar a obesidade, não é capaz de distinguir massa magra de massa adiposa, diferenciar a distribuição de gordura corporal ou detectar o aumento de gordura, podendo ser menos preciso em indivíduos mais idosos, em decorrência da perda de massa magra e diminuição do peso, e superestimado em indivíduos musculosos. ${ }^{18}$ Ao analisarmos os valores obtidos com a mensuração da CC, constatou-se a prevalência da classificação de não obesos, sendo este apuramento antagônico ao obtido nos estudos transversais realizados com moradores da comunidade quilombola de Codó, no Maranhão, em período de tempo diferente. ${ }^{16,19}$

Ao se analisar o estilo de vida, os resultados foram satisfatórios. Houve maior abrangência de participantes que nunca consumiram bebidas alcoólicas e que não eram fumantes, equivalente ao apuramento obtido no estudo realizado com a comunidade quilombola de Alcântara, estado do Maranhão, o qual demonstrou predomínio de 75\% de não etilistas e $77 \%$ de não fumantes. ${ }^{20}$ Contudo, a maior parte da literatura se expressa contraditória, mostrando que o consumo de bebidas alcoólicas e o uso do tabaco é elevado nesse tipo de população. .,10,14,21 $^{2}$ Em continuidade, observou-se que a prática de algum tipo de exercício físico apresentou-se de 
13 | Araújo DAM, Moura TVC, Júnior DNV, Neto FJC, Júnior JSA, Silva ARV

forma positiva. Ao investigar essa variável, levou-se em consideração o tipo de trabalho realizado pelo participante, visto que a maioria da comunidade possui como subsistência a prática da agricultura, o que exige esforço físico e deambulação.

Quanto aos hábitos alimentares, identificou-se autoconsumo de frutas, legumes e hortaliças. Resultado análogo a estudo transversal realizado com indivíduos adultos oriundos de 10 comunidades quilombolas da Bahia, o qual encontrou percentual de $77,8 \%$ para o consumo de frutas e $81,4 \%$ para legumes e hortaliças. ${ }^{22} \mathrm{Da}$ mesma forma, obteve-se altos percentuais de ultraprocessados, como bolacha/biscoito e refrigerantes, os quais são ricos em açúcar, óleos, gorduras e substâncias sintetizadas em laboratório a partir de alimentos e de outras fontes orgânicas, constituindo-se em fator de risco para o desenvolvimento de DCNT. ${ }^{18}$

Quanto ao consumo de embutidos ou enlatados, os resultados foram positivos, demonstrando que a maioria referiu não consumir esse tipo de alimento. Em contrapartida, o consumo de produtos processados, como os salgados fritos foi elevado. Esse tipo de alimento é produzido adicionando sal, açúcar, óleo ou vinagre, os quais são prejudiciais à saúde quando em excesso. ${ }^{18}$

O uso de temperos industrializados mostrou-se elevado; em contraposto, estudo realizado com 213 indivíduos da Comunidade Quilombola Boqueirão, situada na zona rural do município de Vitória da Conquista, no Estado da Bahia, obteve baixo percentual para o uso, tendo maior preferência por temperos caseiros. ${ }^{6}$ Tal resultado obtido na amostra chama a atenção, visto que esse tipo de produto é rico em sódio, cujo consumo excessivo torna-se um dos principais fatores de risco para HA, associando-se a eventos cardiovasculares e renais. ${ }^{8}$

Apesar do elevado consumo de temperos industrializados, os participantes afirmaram não acrescentar sal à comida pronta, o que acaba sendo uma variável favorável para o não desenvolvimento de HA. Entretanto, não se pode fazer uso de generalização para demais comunidades brasileiras, pois ao estudar os quilombolas do Estado de Sergipe obteve-se uma resposta negativa para a pergunta "adiciona sal à comida servida" ${ }^{10}$ No que concerne às 
Fatores associados ao desenvolvimento de hipertensão arterial em uma comunidade qui... | 14

recomendações sobre alimentação saudável, a maioria afirmou nunca ter recebido esse tipo de orientação, diferentemente de estudo transversal realizado com adultos oriundos de 10 comunidades quilombolas do sudoeste da Bahia, o qual identificou que $67,0 \%$ dos participantes afirmaram ter recebido orientações sobre alimentação saudável. ${ }^{22}$

No que diz respeito a visita regular ao serviço de saúde, estudos tiveram seguimento contraditório ao encontrado nesta pesquisa. ${ }^{22-23}$ Entretanto, essa comparação é eivada de vício, uma vez que se deve considerar a realidade a qual está submetida a comunidade em questão, cuja localização geográfica é predominantemente rural, sendo distante da Unidade Básica de Saúde (UBS) que atende a essa região, além da falta de transporte público e particular para o deslocamento até a UBS.

Analisou-se estatisticamente a associação entre os valores da PA com faixa etária, obtendo significância estatística. Da mesma maneira, um estudo observacional realizado na comunidade Mata Cavalo, em Minas Gerais, obteve prevalência de HA nas faixas etárias acima de 40 anos. $^{13}$ Pesquisadores afirmam que à medida que a população quilombola envelhece, o número de hipertensos tende a aumentar. ${ }^{10}$

Analisou-se a associação entre a variável "possui HA” com as variáveis relacionadas ao “estilo de vida e alimentação” dos participantes, havendo significância com a variável "recomendações sobre a alimentação", o que evidencia que a ocorrência de um nível insatisfatório de assistência e a baixa procura pelos serviços de saúde pode favorecer o surgimento de várias doenças, por exemplo, a HA. Observou-se, também, que o resultado obtido da correlação, entre média da pressão arterial sistólica e diastólica com o IMC e CC, foi correspondente aos encontrados em quilombolas do médio São Francisco baiano. ${ }^{9}$

Encontrou-se significância estatística ao correlacionar a média da pressão arterial sistólica e diastólica com o IMC e CC dos participantes, assim como identificado em transversal realizado com populações negras no município de Salvaterra, na Ilha do Marajó. O mesmo 
15 | Araújo DAM, Moura TVC, Júnior DNV, Neto FJC, Júnior JSA, Silva ARV

constatou que as pressões sistólica e diastólica obtiveram uma fraca, porém significante correlação positiva com a circunferência da cintura, demonstrando que a pressão arterial cresce simultaneamente de forma fraca, com a medida da Circunferência da Cintura. ${ }^{12}$

Neste estudo, também analisou-se a associação estatística entre variáveis socioeconômicas e sociodemográficas com a PASM e a PADM, obtendo associações significativas condizentes com os achados literários, os quais demostram que o sexo e renda são fatores determinantes e condicionantes para o desenvolvimento da HA, assim como expuseram um maior percentual de hipertensos casados. ${ }^{5,11,14}$ A promoção do autocuidado por meio da educação em saúde é uma ferramenta fundamental no controle da HAS e/ou Diabetes Mellitus (DM) e os profissionais de saúde precisam buscar ações junto à comunidade no intuito de conhecer, identificar suas dificuldades e planejar intervenções a partir das necessidades daquela realidade. ${ }^{24}$

Por fim, algumas limitações precisam ser consideradas. A primeira está relacionada ao caráter transversal, o que impossibilita o estabelecimento de relações causais por não evidenciar a existência de uma sequência temporal entre exposição ao fator e posteriormente o desenvolvimento da doença. Outra limitação diz respeito a escassez de estudos sobre a temática, dificultando a comparação dos resultados.

\section{Conclusão}

Os fatores associados ao desenvolvimento de HA na comunidade quilombola estudada foram a baixa escolaridade e renda, o alto consumo de temperos industrializados, bolachas/biscoitos, salgados fritos e refrigerantes, bem como, o não recebimento de orientações nutricional e a baixa procura pelos serviços de saúde.

Os seguimentos obtidos podem viabilizar intervenções direcionadas as reais necessidades dos indivíduos desta comunidade. Estudos dessa natureza contribuem como medida preventiva 
Fatores associados ao desenvolvimento de hipertensão arterial em uma comunidade qui... | 16

facilitando o controle de doenças crônicas. Portanto, busca-se um engajamento das lideranças das comunidades, a fim de pleitear junto ao poder público na obtenção de políticas públicas.

Da mesma forma, anseia-se por mudança na filosofia de trabalho da equipe da ESF no intuito de planejar e expandir ações de educação em saúde buscando atender as necessidades dos moradores que residem nessa localidade. Nessa mesma linha, incentivar a realização de visitas domiciliares promovendo assim, maior continuidade da atenção à saúde e possibilitando uma criação de vínculo.

\section{Referências}

1. Machado JC, Cotta RMM, Moreira TR, Silva LS. Análise de três estratégias de educação em saúde para portadores de hipertensão arterial. Ciênc Saúde Colet. 2016;21(2):611-20. doi: https://doi.org/10.1590/141381232015212.20112014

2. Malta DC, Bernal RTI, Andrade SSCA, Silva MMA, Velasquez-Melendez G. Prevalência e fatores associados com hipertensão arterial autorreferida em adultos brasileiros. Rev Saúde Pública. 2017; 51(Suppl 1):1-11. doi: 10.1590/s1518-8787.2017051000006

3. Belfort IKP, Avelar MF, Nunes JDC, Monteiro SCM. Elevação de níveis pressórios em uma comunidade quilombola. Rev Bras Promoç Saúde. 2017;30(3):1-8. doi: https://doi.org/10.5020/18061230.2017.6135

4. Barreto NDM, Pacheco JRB, Marins SR, Magalhães CF, Cardoso GP, Houaiss M. Prevalência da hipertensão arterial nos indivíduos de raça negra. Arq Bras Med. 1993;67(6):449-51.

5. Bezerra VM, Andrade ACS, Medeiros DS, Caiaffa WT. Pré-hipertensão arterial em comunidades quilombolas do sudoeste da Bahia, Brasil. Cad Saúde Pública. 2017;33(10):e00139516. doi: https://doi.org/10.1590/0102-311x00139516

6. Silva TSS, Bonfim CA, Leite TCR, Moura CS, Belo NO, Tomazi, L. Hipertensão arterial e fatores associados em uma comunidade quilombola da Bahia, Brasil. Cad Saúde Colet. 2016;24(3):376-83. doi: https://doi.org/10.1590/1414-462x201600030068

7. Ministério da Saúde (BR). Orientações para coleta e análise de dados antropométricos em serviços de saúde: norma técnica do sistema de Vigilância Alimentar e Nutricional - SISVAN. Brasília (DF): Ministério da Saúde; 2011.

8. Malachias MVB, Póvoa RMS, Nogueira AR, Souza D, Costa LS, Magalhães ME, et al. 7th Brazilian guideline of arterial hypertension. Arq Bras Cardiol. 2016;107(Supl 3):1-83. doi: 
https://doi.org/10.5935/abc.20160153

9. Mussi RFF, Queiroz BM, Petróski EL. Excesso de peso e fatores associados em quilombolas do médio São Francisco baiano, Brasil. Ciênc Saúde Colet. 2018;23(4):1193-200. doi: https://doi.org/10.1590/141381232018234.03662016

10. Santos DMS, Prado BS, Oliveira CCC, Almeida-Santos MA. Prevalência da hipertensão arterial sistêmica em comunidades quilombolas do estado de Sergipe, Brasil. Arq Bras Cardiol. 2019;113(3):38390. doi: https://doi.org/10.5935/abc.20190143

11. Santos RC, Silva MS. Condições de vida e itinerários terapêuticos de quilombolas de Goiás. Saúde Soc. 2014;23(3):1049-63. doi: https://doi.org/10.1590/S0104-12902014000300025

12. Cordovil YF, Almeida SS. Variáveis antropométricas e fatores de risco cardiovascular associados em Quilombolas Marajoaras. RBONE [Internet]. 2018 nov [acesso em 2019 nov 21];12(71):406-15. Disponível em: http://www.rbone.com.br/index.php/rbone/article/view/718

13. Santos EC, Scala LCN, Silva AC. Prevalência de hipertensão arterial e fatores de risco em remanescentes de quilombos, Mato Grosso, Brasil. Rev Bras Hipertens [Internet]. 2015 dez [acesso em 2019 dez 01];22(3):1005. Disponível em: http://docs.bvsalud.org/biblioref/2018/03/881238/rbh_v22n3_100-105.pdf

14. Melo MFT, Silva HP. Doenças crônicas e os determinantes sociais da saúde em comunidades quilombolas do Pará, Amazônia, Brasil. Rev ABPN [Internet]. 2015 [acesso em 2019 dez 15];7(16):168-89. Disponível em: https://www.abpnrevista.org.br/index.php/site/article/view/103

15. Pauli S, Bairros FS, Nunes LN, Neutzling MB. Prevalência autorreferida de hipertensão e fatores associados em comunidades quilombolas do Rio Grande do Sul, Brasil. Ciênc Saúde Colet. 2019; 24(9):3293-303. doi: https://doi.org/10.1590/1413-81232018249.28002017

16. Castro, M. M. S. Hipertensão arterial e fatores associados: um estudo em comunidades de afrodescendentes quilombolas no maranhão [monografia]. São Luís: Universidade Federal do Maranhão; 2015. Curso de Medicina.

17. Mariosa DF, Ferraz RRN, Santos-Silva EN. Influência das condições socioambientais na prevalência de hipertensão arterial sistêmica em duas comunidades ribeirinhas da Amazônia, Brasil. Ciênc Saúde Colet. 2018;23(5):1425-36. doi: https://doi.org/10.1590/1413-81232018235.20362016

18. Associação Brasileira para o Estudo da Obesidade e da Síndrome Metabólica. Diretrizes brasileiras de obesidade 2016. 4ª ed. São Paulo: Associação Brasileira para o Estudo da Obesidade e da Síndrome Metabólica; 2016.

19. Fonseca RLM. Dislipidemia em comunidades de afrodescendentes quilombolas no Maranhão [monografia]. São Luís: Universidade Federal do Maranhão; 2016. Curso de Medicina. 
Fatores associados ao desenvolvimento de hipertensão arterial em uma comunidade qui... | 18

20. Silva CM. Avaliação da gordura visceral por tomografia computadorizada em hipertensos de população quilombola de Alcântara-MA [monografia]. São Luís: Universidade Federal do Maranhão; 2017. Curso de Medicina.

21. Freitas IA, Rodrigues ILA, Silva IFS, Nogueira LMV. Perfil sociodemográfico e epidemiológico de uma comunidade quilombola na Amazônia Brasileira. Rev Cuid. 2018;9(2):2187-200. doi: https://doi.org/10.15649/cuidarte.v9i2.521

22. Bezerra VM, Caiaffa WT, Andrade ACS, César CC. Desconhecimento da hipertensão arterial e seus determinantes em quilombolas do sudoeste da Bahia, Brasil. Ciênc Saúde Colet. 2015;20(3):797-807. doi: https://doi.org/10.1590/1413-81232015203.14342014

23. Andrade AO, Aguiar MIF, Almeida PC, Chaves ES, Araújo NVSS, Freitas Neto JB. Prevalência da hipertensão arterial e fatores associados em idosos. Rev Bras Promoç Saúde [Internet]. 2015 [acesso em 2019 nov 21];27(3):303-11. Disponível em: https://periodicos.unifor.br/RBPS/article/view/2729

24. Oliveira MSN, Almeida GBS, Chagas DNP, Salazar PR, Ferreira LV. Autocuidado de idosos diagnosticados com hipertensão arterial e/ou diabetes mellitus. Rev Enferm UFSM. 2017;7(3): 490-503. doi: https://doi.org/10.5902/2179769226344

Editora Científica: Tânia Solange Bosi de Souza Magnago

Editora Associada: Rosângela Marion da Silva

\author{
Autor correspondente \\ Dinah Alencar Melo Araújo \\ E-mail: jcamo@hotmail.com \\ Endereço: Rua São Sebastião, 653, Malva, Picos-PI. \\ CEP: 64083-030
}

\title{
Contribuições de Autoria
}

\section{1 - Dinah Alencar Melo Araujo}

Concepção ou desenho do estudo/pesquisa, análise e/ou interpretação dos dados, revisão final com participação crítica e intelectual no manuscrito.

\section{2 - Tatiana Victoria Carneiro Moura}

Concepção ou desenho do estudo/pesquisa, análise e/ou interpretação dos dados, revisão final com participação crítica e intelectual no manuscrito.

\section{3 - Denival Nascimento Vieira Júnior}

Concepção ou desenho do estudo/pesquisa. 
19 | Araújo DAM, Moura TVC, Júnior DNV, Neto FJC, Júnior JSA, Silva ARV

\section{4 - Francisco João de Carvalho Neto}

Concepção ou desenho do estudo/pesquisa.

\section{5 - José de Siqueira Amorim Júnior}

Revisão final com participação crítica e intelectual no manuscrito.

\section{6 - Ana Roberta Vilarouca da Silva}

Concepção ou desenho do estudo/pesquisa, análise e/ou interpretação dos dados, revisão final com participação crítica e intelectual no manuscrito.

\section{Como citar este artigo}

Araújo DAM, Moura TVC, Júnior DNV, Neto FJC, Júnior JSA, Silva ARV. Fatores associados ao desenvolvimento de hipertensão arterial em uma comunidade quilombola. Rev. Enferm. UFSM. 2021 [Acesso em: Ano Mês Dia]; vol.11 e33: 1-19. DOI:https://doi.org/10.5902/2179769248318 